\title{
Desafíos para las generaciones en la educación superior en salud
}

\section{Challenges for generations in higher education in health}

\author{
Santiago Patiño-Giraldo • Medellín (Colombia)
}

DOI: https://doi.org/10.36104/amc.2020.1649

\section{Resumen}

Las características de uso de tecnologías de la información y la comunicación en la sociedad actual generan una serie de desafíos en los escenarios de aprendizaje para la educación superior en salud. Este artículo tiene como objetivo presentar varios casos problemáticos que dan cuenta de estos desafíos y propuestas de cómo manejarlos. El principal elemento con el que cuentan los profesores en educación superior en salud es el establecimiento de reglas y límites claros en el relacionamiento con las nuevas generaciones. Unos escenarios de aprendizaje que hagan uso de las tecnologías de la información en la comunicación, en un ambiente de confianza y emocionalmente seguros deberían ser el objetivo de los currículos en educación superior en salud. (Acta Med Colomb 2020; 45. DOI: https://doi.org/10.36104/amc.2020.1649).

Palabras clave: educación médica, aplicaciones de la informática médica, educación a distancia .

\begin{abstract}
The characteristics of information and communication technology use in today's society create a series of challenges within health sciences higher education learning scenarios. The objective of this article is to present several problematic cases which illustrate these challenges and propose ways to handle them. The main element at the disposal of health sciences higher education professors is the establishment of rules and clear limits in the interaction with the new generations. Learning scenarios in which information technologies for communication are used in an emotionally safe atmosphere of trust should be the goal of curricula in health sciences higher education. (Acta Med Colomb 2020; 45. DOI: https://doi.org/10.36104/amc.2020.1649).

Key words: medical education, applications of medical informatics, distance learning.
\end{abstract}

Dr. Santiago Patiño-Giraldo: Profesor Departamento de Medicina Interna, Universidad de Antioquia. Internista Sección Medicina Interna, Hospital Pablo Tobón Uribe. Medellín (Colombia).

Correspondencia: Dr. Santiago Patiño-Giraldo. Medellín (Colombia).

E-mail: Santiago.patino@udea.edu.co Recibido: 22/I/2020Aceptado: 19/II/2020

\section{Introducción}

La educación es un proceso humano y social de transmisión y transformación de la cultura (1). En términos generales, se trata de una relación generacional, donde un grupo de conocimientos se entrega de parte de una de ellas para que sea acogido, criticado y nutrido por la nueva generación. En el contexto formal, es decir el institucional o la escuela, se enseña, siendo los conocimientos específicos mediados por un currículo, buscando un ideal de hombre (2).

Los procesos de enseñanza y de aprendizaje están plasmados en los planes de estudio que, dependiendo del perfil de ser humano que se pretende formar, usa diferentes espacios de enseñanza y aprendizaje. Estos espacios son dinámicos, entre tanto dependen de las conductas que tengan los actores del proceso educativo, es decir de los estudiantes y los profesores, en un primer nivel, de los padres y los administrativos en un segundo nivel y de la sociedad en general en un nivel más amplio (2).

La sociedad actual tiene unas particularidades que originan una serie de retos en los escenarios de aprendizaje incluidos aquellos de la educación superior en salud. Es así como se describen los siguientes desafíos en la educación médica: un sistema de salud en cambio, expectativas de la sociedad diferentes frente a su salud o enfermedad, la seguridad del paciente durante su atención, la ética y el profesionalismo (humanización), los cambios en el énfasis curricular (de objetivos a competencias y ahora a resultados de aprendizaje), la cantidad de información, la necesidad de aprendizaje continúo, los cambios tecnológicos y la nueva generación de estudiantes (3). Este artículo tiene como 
finalidad presentar algunos de los desafíos en la nueva generación de estudiantes en relación con los escenarios de aprendizaje en educación superior en salud para establecer algunas recomendaciones en su manejo.

\section{Conceptualización}

Para comprender el tema en cuestión, es necesario definir los conceptos de desafío, generación y escenario de aprendizaje. La palabra desafío etimológicamente proviene del griego des (negación de acción) y afiar, verbo utilizado en la antigüedad para dar garantía de respeto a la integridad física y bienes de otro (4). Desafiar es entrar en disputa. Según la Real Academia de la Lengua, también se entiende como la acción de enfrentarse a las dificultades con decisión (5). Se entiende entonces en este artículo como desafío al acto consciente del profesor de enfrentarse (valerosamente) a una condición adversa en los escenarios de aprendizaje.

El concepto de generación tiene unas raíces históricas de principios del siglo XX. Se reconoce como una generación aquella que comparte tres características: un periodo de tiempo de nacimiento, 2) un momento histórico (periodo o era) y 3 ) unas condiciones socioculturales (6). En un ejercicio durante un encuentro de educación superior en salud, se validó cuál es el significado que tiene una generación en la comunidad académica (Figura 1). En la sociedad red (7), las generaciones desde el punto de vista temporal son más cortas. Esto sumado al aumento de la expectativa de vida y a la prolongación de los tiempos en la educación institucionalizada, aumentan el número de generaciones que comparten el proceso educativo.

Podría decirse que actualmente confluyen en la educación superior en salud: los baby-boomer (1946-1964), la generación X (1965-1980), los milenials (1980-1996) y los centenials (1996 a la actualidad). Las dos últimas generaciones han sido descritas también como generación "C" - conectados a la red en todo momento- (8), generación app -por su tendencia a resolver sus problemas a través de estos programas informáticos de los teléfonos móviles- (9), generación YouTube -dado su interés por las redes sociales con alto contenido visual- (10) o nativos digitales por su estrecha relación con la tecnología y su subsecuente impacto en el comportamiento (11).

Es posible que el desafío mayor no sean las nuevas generaciones sino la confluencia de cuatro de ellas en los escenarios de aprendizaje en educación superior en salud. Por ejemplo, los pacientes y familiares pueden ser de cualquiera de estas generaciones, los estudiantes son milenials o centenials, los profesores están distribuidos en baby-boomers, generación X y milenials y los administrativos en su gran mayoría pertenecen a la generación más antigua.

Finalmente, los escenarios de aprendizaje se entienden como espacios educativos compuestos por dos dimensiones una psicosocial y otra material. Las dimensiones psicosociales tienen mucha relación con las generaciones y se compone de tres subdominios: el personal (docente-discente), el social y el organizacional (la estructura de la institución educativa).
Por otra parte la dimensión material cuenta con dos subdominios: el espacio real (aulas de clase, hospital, consultorio, comunidad) y el virtual (sistemas de aulas virtuales, redes sociales, entre otros) (12).

\section{Contexto sociocultural de la educación superior en salud}

La educación superior en salud está permeada por la sociedad red, cuya característica principal no es la información per se, puesto que todas las sociedades se han basado en información. Su gran diferencia está en la gran capacidad de transmisión de información y datos así como la conexión permanente que tienen teóricamente todos los seres humanos a las fuentes de información a través de las tecnologías de la información y la comunicación $\operatorname{TIC}(7,13)$.

Son las TIC, y más específicamente la velocidad con la cuál estas tecnologías se actualizan, unos de los factores más determinantes de los desafíos en los escenarios de aprendizaje (14). Según la Ley de Moore, la tecnología duplica su velocidad en forma exponencial (el doble de capacidad a la mitad del precio cada dos años) (15). Lamentablemente, esta velocidad de cambio supera la velocidad de adaptación de la humanidad (que tiene un comportamiento más lineal) explicando la sensación de pérdida, velocidad y desacomodamiento continuo en todas las generaciones (14).

Por otra parte, el sistema de salud ya no exige al personal de salud solo cuidado, acompañamiento o alivio durante el sufrimiento. A esto se han sumado otras exigencias que incluyen: la seguridad del paciente (incluyendo la privacidad de su información personal), una atención en salud de calidad (con las múltiples definiciones de calidad que puedan encontrarse), la reducción de costos en salud y unos desenlaces clínicos relevantes (desde la perspectiva del paciente) todo esto sin que afecte e incluso mejore la calidad de vida de los profesionales de la salud (16).

Este ambiente de cambio (social y del sistema de salud) contrasta con la velocidad a la cual pueden responder los currículos universitarios. Por ejemplo, los registros calificados en Colombia se asignan para un horizonte temporal de siete años (17), periodo en el cual algunas metas en salud o tecnologías se considerarán obsoletas mientras que otras se habrán introducido. De allí la necesidad de que las instituciones de educación superior se replanteen sus objetivos hacia el autoaprendizaje de sus estudiantes y la educación continúa, con cambios sostenibles, a costos razonables sin que exijan mucho espacio-tiempo (14).

En último lugar, si se considera la distribución tradicional de los currículos en cursos básicos, preclínicos, clínicos y comunitario, teniendo en cuenta que en cada uno de ellos se tiene un número plural de profesores en diferentes escenarios de aprendizaje (considerando los distintos centros de práctica), dos estudiantes diferentes tendrán experiencias educativas diversas dependiendo de los escenarios de aprendizaje por lo que discurran lo cual escapa a la homogeneidad que el proyecto educativo de programa (PEP) pretende establecer. 
Es decir, si en el currículo manifiesto ya hay un número importante de retos, en el currículo manifiesto y en el currículo oculto, los posibles desafíos son mucho mayores (18).

\section{Desafíos en educación superior en salud para las generaciones}

Teniendo este contexto, se presentan los desafíos que tienen las generaciones partícipes del proceso de educación en salud. Cada uno de ellos está en una situación que potencialmente se presenta en la educación superior en salud actual.

\section{Exigencia de garantías}

Situación problema. Luego de cuatro semanas de avances en su curso, un estudiante manifiesta: "Hola profe: el grupo estuvo revisando el decreto 2566 donde reza que un crédito académico corresponde a 48 horas de trabajo. Vemos con preocupación que su materia tiene dos créditos y en sólo cuatro semanas hemos dedicado 40 horas, por lo que le solicitamos ajustes al tiempo porque sólo deberíamos trabajar dos horas a la semana y no diez."

El estudiante de las generaciones milenial y centenial, entiende la educación como un servicio por el que se está pagando y por tanto exigen que se cumplan los términos del contrato educativo (currículo y plan de estudios) (8). No es extraño que vayan al espacio académico con el microcurrículo del curso para verificar el cumplimiento de lo registrado en su contenido, distribución y carga horaria. Exigen que se haga lo que está escrito en dichos documentos.

Además, piensa que sus calificaciones son reflejo de la opinión y relación que tiene con su docente y no de su desempeño académico. Esto se debe al hecho de que fueron criados en un ambiente en el cual se es merecedor a un premio por el solo hecho de participar en una actividad particular y no por su real desempeño, lo que han denominado algunos como la generación de los trofeos (19). Es una generación que fue criada con el pensamiento de "todo lo puedes", por lo que las circunstancias que se manifiesten como incapacidades de alto logro y desempeño (por ejemplo, bajas calificaciones) generan ansiedad.

Para enfrentar este desafío hay que ser muy explícito en las reglas del juego al comienzo del curso, aproximándose a lo descrito en los microcurrículos y advirtiendo que es posible que algunas situaciones varíen por diferentes circunstancias. También es importante definir cuáles son las expectativas del docente durante el desarrollo del curso en su dedicación horaria, esfuerzo, cumplimiento de tiempos de tareas, entre otros. Esto debe contrastarse con las expectativas de los discentes teniendo como resultado intereses comunes entre docente y estudiante $(8,20)$.

\section{Relacionamiento horizontal \\ Situación problema: Durante ronda clínica un es- tudiante lo aborda y le dice: "Hola Pedro, ¿Cómo}

vas? Me enteré de que estás con mucho trabajo, te veo estresado con eso, ¿te ayudo?"

La crianza de los estudiantes de las nuevas generaciones se caracterizó por un relacionamiento horizontal y de amistad con sus padres $(21,22)$. Esperan que todas sus interacciones con la autoridad se desarrollen de la misma forma. Dirigirse al docente por su nombre de pila es la regla, haciendo poco uso de los títulos (doctor, don, profesor). A su vez pueden sentirse extraños cuando se dirigen de esa forma hacia ellos. Quieren que el profesor sea amigable y que se compartan espacios con ellos más allá de los académicos. Tienen gracias a las redes sociales la posibilidad de conocer más esferas de la vida privada de sus profesores, lo cual era muy difícil en el pasado (8).

Entendiendo su modo de ver a la autoridad como un par, es comprensible que pretendan que todo sea negociable: la distribución de las cargas académicas, las calificaciones, los horarios y todo lo relacionado en el "contrato" educativo.

Se recomienda frente a este tipo de relacionamiento ser tolerante así no se comparta esta forma de comunicarse y comprender que es natural para el estudiante no utilizar los formalismos y que no es signo de irrespeto. Es muy importante frente a las relaciones extracurriculares establecer fronteras de parte del profesor siendo explícito hasta donde puede el discente acceder a información privada de aquél. Aceptar invitaciones de "amistad" en redes sociales, o "seguir" en las redes entre estudiantes y profesores debe ser consecuente con estas reglas explícitas (8).

\section{Pragmatismo participativo}

Situación problema. En búsqueda de nuevos escenarios de aprendizaje se desarrolla un aula inversa. Al llegar al espacio de discusión usted inicia preguntando: ¿cuál es la fisiopatología que vieron de la enfermedad en la video clase? Pero nadie responde.

El aula inversa es una estrategia didáctica en la cual las actividades magistrales que tradicionalmente se llevan a cabo en el escenario de aprendizaje presencial se trasladan al hogar para aprovechar el tiempo con el profesor avanzando en la aplicación de la teoría (de ahí su calificativo de inversa) (23). Si bien esto puede traducirse en una optimización del uso de tiempo de docente y discente, en personalización de los contenidos y en uso de las TIC en una generación acostumbrada a ellas, puede encontrarse que los estudiantes no revisen los contenidos antes de llegar al aula, porque el currículo no aporta espacios de tiempo independiente suficiente para ello (24) perdiendo todo el beneficio y generando malestar particularmente en el profesor.

Por otra parte, las nuevas generaciones si bien exigen tener voz y voto en los escenarios de aprendizaje, prefieren criticar a ser criticados, por tanto, las preguntas que puedan exponerlos frente a sus compañeros tenderán al fracaso (como la utilizada en la situación problema). Preguntas como “¿qué hubieran hecho distinto en ese caso? o ¿en qué creen que el autor de ese texto actuó en forma correcta?" cambian 


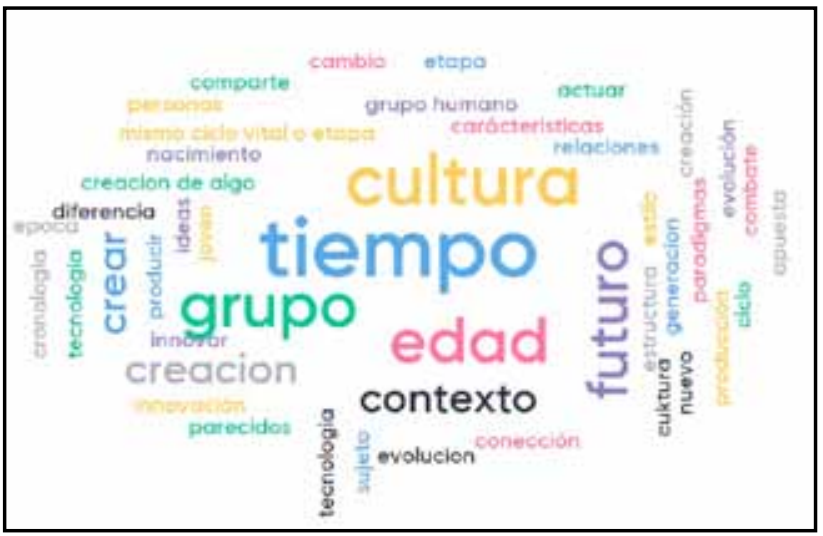

Figura 1. Nube de palabras del concepto de generación construido durante sesión magistral de desafíos de educación en salud para las nuevas generaciones.

el foco de crítica hacia un externo y facilita la participación en el aula inversa (8).

Como característica adjunta, el desarrollo de motores de búsqueda y las aplicaciones móviles, generan en los estudiantes una menor dependencia a su memoria ya trasladada a dichos dispositivos, buscando una respuesta oportuna, entiéndase como inmediata, como la que ofrecen estos desarrollos tecnológicos. Preguntas que no tengan claramente una aplicación práctica suelen ser desestimadas por las nuevas generaciones (25).

En conclusión, los escenarios de aprendizaje están mediados por el grado de crítica al que los estudiantes puedan sentirse expuestos y de la pertinencia (desde lo práctico y funcional) de las preguntas que se realicen las cuales se buscarán responder inmediatamente a través de sus dispositivos electrónicos conectados a la red en la mayoría de los casos.

Enfrentarse a este reto es complejo. Impedir el uso de los teléfonos móviles es una salida que parece tentadora, pero en términos sociales es imposible de lograr. Nuevamente el establecer fronteras de que se espera sea resuelto con y sin ayuda de sus dispositivos electrónicos, abrir espacios curriculares para las actividades independientes y relacionar las preguntas con los problemas presentes y futuros que enfrentarán los estudiantes es la mejor forma de salir avante en esta situación (8).

\section{Generación multitarea}

Situación problema. De un escenario de práctica clínica reportan la inasistencia y falta de respuesta de uno de los estudiantes. Usted cita al estudiante, pero éste no asiste a la oficina. Dos días después le busca en un pasillo y le dice que está haciendo muchas obligaciones extracurriculares y por eso no fue.

Como se discutió previamente, las relaciones horizontales son la regla para las nuevas generaciones. Los escenarios formales, como las oficinas o las citas programadas para ser evaluados generan un estado de ansiedad que las dificulta (8). Cuando se llevan a cabo quieren un lenguaje positivo y esperan que se entienda que, bajo las condiciones de sociedad actual, ellos tienen muchas más cosas en qué pensar y no sólo en la academia. Es una generación multitarea que quiere ser reconocida como tal.

$\mathrm{Si}$ bien se percibe una indiferencia hacia la autoridad, hay que reconocer que es la primera generación que no requiere estrictamente de un superior para acceder a la información. No es extraño que los estudiantes se pregunten que puede ofrecer un docente que no esté en internet y los profesores deben estar preparados para hacer frente a esta pregunta $(8,20)$.

El éxito fácil que alcanzan figuras de la edad del estudiante (youtubers o influenciadores), asociado a la costumbre de recibir estímulo por acciones que en generaciones previas se consideraban mínimas, producen expectativas poco realistas frente al resultado de su esfuerzo académico. Pueden esperar la excelencia a pesar de no asistir a los escenarios de aprendizaje, o culpar a la prueba antes que considerar a su nivel de esfuerzo para alcanzar los objetivos (8).

Nuevamente, establecer expectativas claras, definir en las escalas de evaluación cuál es el esfuerzo esperado para alcanzar el éxito académico, establecer comunicación con los padres de familia, en conclusión, definir un diálogo frecuente y sensato entre docentes y discentes es la forma de enfrentarse a este desafío.

\section{Integración de las TIC}

Situación problema. Dentro de los planes de desarrollo institucional se resalta el uso de las TIC en el currículo. Todos los profesores tienen un ambiente virtual de aprendizaje pero no es mas que un repositorio de documentos con pobre interacción entre docentes y estudiantes.

La introducción de las TIC en la educación superior en salud debe redefinir mas no sustituir los ambientes de aprendizaje. El solo hecho de que se esté inmerso en una sociedad hiperconectada no garantiza el éxito del uso de las TIC en educación (24). De hecho, el uso de ellas trae consigo nuevos retos que se agrupan en cuatro categorías: flexibilidad, motivar/interacción, facilitar proceso de aprendizaje y clima de aprendizaje afectivo (28).

Primero, la flexibilidad es una de las características principales de la educación virtual y se puede evidenciar en el espacio (no se requiere de uno físico para el encuentro docente-discente), el tiempo (desarrollo de actividades asincrónicas en el momento que el estudiante lo requiera), vía (desarrollo de contenidos en el orden que el estudiante lo vea conveniente) y cadencia (seguimiento de los contenidos a la velocidad que el estudiante lo quiera). La mayoría de los desarrollos en educación virtual limitan la flexibilidad a la espacial, pues las fechas de entrega y los contenidos suelen venir predefinidos por el docente (28).

Segundo, la motivación-interacción, se logra a través de actividades que establezcan diálogos en tres vías: docenteestudiante, estudiante-estudiante y docente-docente. Las 
redes de comunicación virtuales son mejores para sostener relaciones que para crearlas, por lo que un encuentro presencial donde se conozcan profesores y estudiantes es la mejor forma de facilitar la interacción futura en ambientes virtuales de aprendizaje (28).

En tercer lugar, para facilitar el proceso de aprendizaje, el profesor debe desarrollar habilidades no técnicas en el estudiante tales como: organización, disciplina, manejo del tiempo, habilidades tecnológicas y capacidad de autoevaluación (28). Por supuesto, se debe orientar y planear el escenario de aprendizaje virtual estableciendo una evaluación de conocimientos previos, contando con la información organizacional (plan educativo institucional, proyecto educativo de programa, currículo, plan de estudios), informando las expectativas a los estudiantes y familiarizando a los estudiantes con la tecnología a utilizar durante el ambiente virtual de aprendizaje. $\mathrm{Al}$ final, esta planeación requiere de una monitorización continúa haciendo uso de: 1) actividades por pares, 2) seguimiento a los indicadores de uso de la plataforma, 3) evaluación formativa y 4) la comunicación por medios sincrónicos y asincrónicos (correo electrónico, reportes, estadísticas).

Para terminar, el clima emocional, hace parte del paradigma actual de la World Wide Web, donde los sentimientos y la forma de transmitirlos se vuelven el centro (web 5.0). La comunicación empática, con adecuado uso del sentido del humor, los mensajes frecuentes de aliento en lenguaje positivo para dirigir la atención a los aspectos más relevantes del curso son las formas descritas de generar un buen clima emocional. Lastimosamente, pocos estudios indican cómo trabajar con las emociones en ambientes virtuales de aprendizaje (28).

\section{Coda: El síndrome de quemado (burnout) en los estudiantes de medicina}

El síndrome de quemado es un constructo multivariable caracterizado por varios grados de desgaste emocional, despersonalización (sentimiento de indiferencia hacia los pacientes) y baja percepción de desarrollo personal. Esta situación trae como consecuencias disminución de la empatía, aumento de fraude, deserción estudiantil, errores médicos e ideación suicida (27).

Estudios en los Estados Unidos, demuestran que aproximadamente $50 \%$ de los estudiantes de pre y posgrado presentan el síndrome, principalmente el constructo de despersonalización (27). Es interesante reconocer que los ambientes de aprendizaje en salud (particularmente medicina) inciden en el desarrollo del síndrome. Esto se ha demostrado en estudios comparativos de cohortes de la misma edad y desarrollo escolar que escogen diferentes profesiones.

Los factores de los escenarios de aprendizaje que se asocian al síndrome son una relación directamente proporcional con el grado de competición en el que se desarrolle el currículo (en términos de calificaciones principalmente) e inversamente proporcional al grado de trabajo colabora-

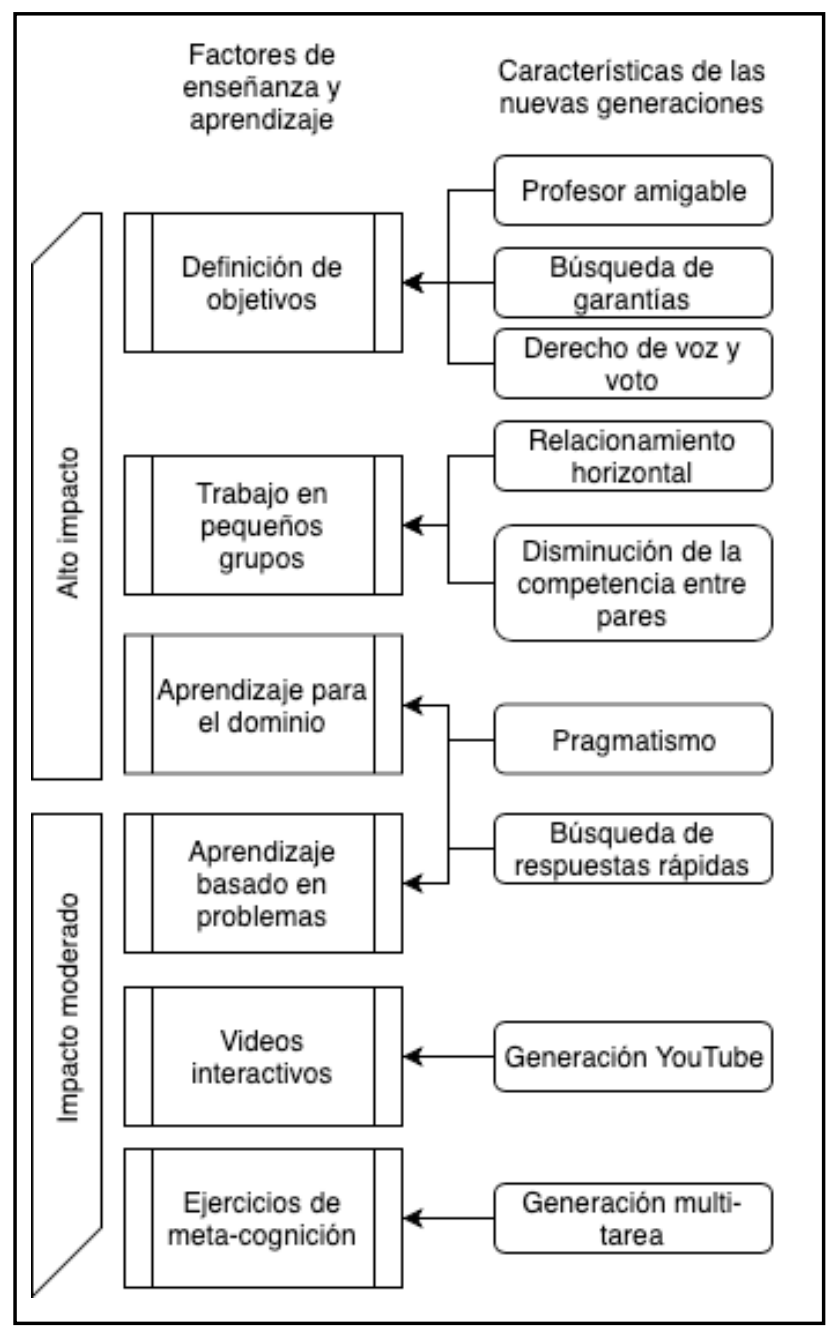

Figura 2. Relación entre factores de enseñanza de impacto alto y moderado y las características de las nuevas generaciones.

tivo, la calidad docente y la organización de las escenarios clínicos. A pesar de que se ha asociado a la carga laboral elevada, en los estudiantes no se encuentra asociación con el número de pacientes, su complejidad, las horas de actividad ni al tiempo de vacaciones (27).

Frente a este desafío podrían considerarse sistemas de evaluación menos competitivos (pasar de escalas numéricas a escalas dicotómicas), programas de tutores pares, programas de bienestar universitario fuertes, didácticas de trabajo en grupo y rotaciones organizadas longitudinalmente que permitan un mejor seguimiento y relación entre docente y discente.

\section{¿Vale la pena desafiar a los escenarios de aprendizaje para las nuevas generaciones?}

La investigación en educación superior en salud valida el efecto positivo en los resultados de aprendizaje de enfrentar los retos anteriormente descritos (Figura 2). Huang y colaboradores (29), en un metaanálisis de estudios controlados de asignación aleatoria y cuasi experimentales (pre-pos) 
identificaron que los factores de enseñanza y aprendizaje que más efecto tienen son el aprendizaje para el dominio, el trabajo en pequeños grupos y la definición de objetivos, seguidos del uso de videos interactivos (30), el aprendizaje basado en problemas y los ejercicios de metacognición.

El trabajo colaborativo, no sólo entre estudiantes, sino entre las diferentes áreas de la salud (transdisciplinariedad) debería ser un paradigma para innovar y enfrentarse a los desafíos actuales en educación superior en salud (31). Para evaluar un escenario de aprendizaje frente a estos retos habrá que preguntarse si: es seguro para los pacientes, influye positivamente en la calidad del cuidado en salud, facilita la transición del cuidado, tiene supervisión directa por el docente, presenta planes para manejar y mitigar la fatiga y facilita el desarrollo de habilidades no técnicas (profesionalismo) $(32,33)$. Los administradores en educación superior en salud deberán estar atentos en los comités docencia servicio de estas dimensiones para dar continuidad o no a los escenarios de práctica clínicos.

\section{Conclusión}

El impacto de las tecnologías de la información y la comunicación en la sociedad red y las nuevas modalidades de enseñanza y aprendizaje, trajeron consigo la confluencia de cuatro distintas generaciones a los escenarios de aprendizaje con desafíos para cada una de ellas. Mientras los estudiantes están aprendiendo una disciplina, los administradores de educación y los profesores aprenden la mejor forma de enseñarla. Un escenario de aprendizaje para estas generaciones debería ser bimodal (presencial y virtual), con reglas claras explícitas desde el principio, pero abiertas al cambio según la voz y voto de los estudiantes, dirigida al aprendizaje situado (significativo) y emocionalmente seguro para todos.

\section{Referencias}

1. González Agudelo EM. Corrientes pedagógicas contemporáneas. Medellín Univ Antioquia. 1999;

2. González Agudelo EM, de ZAYAS A, Carlos M. Lecciones de didáctica general. Magisterio Bogotá. 1998;121.

3. Guze PA. Using technology to meet the challenges of medical education. Trans Am Clin Climatol Assoc. 2015;126:260.

4. Monlau PF. Diccionario etimológico de la lengue castellana: (ensayo) precedido de unos rudimentos de etimología. M. Rivadeneyra; 1856.578 p.

5. Española RA. Diccionario de la lengua española, [versión 23.2 en línea]. Consult En Httpsdle Rae Es. 2018;

6. Pilcher J. Mannheim's Sociology of Generations: An Undervalued Legacy. Br J Sociol. 1994;45(3):481-494.

7. Castells M, Schmalenberger M. La sociedad red: una visión global. Madrid: Alianza Editorial; 2006.
8. Espinoza C. Millennial values and boundaries in the classroom. New Dir Teach Learn. 2012;2012(131):29-41.

9. Gardner H, Davis K. La generación APP. Cómo Los Jóvenes Gest Su Identidad Su Privacidad Su Imagin En El Mundo Digit Bogotá Planeta. 2014;

10. Barry DS, Marzouk F, Chulak-Oglu K, Bennett D, Tierney P, O'Keeffe GW. Anatomy education for the YouTube generation. Anat Sci Educ. 2016;9(1):90-96.

11. Prensky M. Nativos e inmigrantes digitales. Distribuidora Sek; 2010.

12. Padmore JS. A conceptual framework of the clinical learning environment in medical education [PhD Thesis]. University of Maryland University College; 2015.

13. Castells M, Tubella I, Sancho T, Roca M. La transición a la sociedad red [Internet]. 1a ed. Barcelona: Editorial Ariel; 2007 [citado el 15 de enero de 2017]. 238 p. Disponible en: https://dialnet.unirioja.es/servlet/libro?codigo=358484

14. Friedman TL. Gracias por llegar tarde. Barcelona, Deusto; 2018.

15. Moore G. Moore's law. Electron Mag. 1965;38(8):114.

16. Mohamed AL. Future Challenges in Medical Education. Bangladesh J Med Sci. enero de 2010;9(1):4-13

17. Ministerio de Educación Nacional. Decreto 1330 de julio 25 de 2019 - Por el cual se sustituye el Capítulo 2 y se suprime el Capítulo 7 del Título 3 de la Parte 5 del Libro 2 del Decreto 1075 de 2015 -Único Reglamentario del Sector Educación [Internet]. 1330 jul 25, 2019. Disponible en: https://www.mineducacion. gov.co/1759/w3-article-387348.html?_noredirect=1

18. Forero DA, Majeed MH, Ruiz-Díaz P. Current trends and future perspectives for medical education in Colombia. Med Teach. el 6 de septiembre de 2019;1-7.

19. Tulgan B. Not everyone gets a trophy: How to manage generation Y. John Wiley \& Sons; 2009

20. Eckleberry-Hunt J, Tucciarone J. The challenges and opportunities of teaching "Generation Y". J Grad Med Educ. 2011;3(4):458-461.

21. Deal JJ, Stawiski S, Graves LM, Gentry WA, Ruderman M, Weber TJ. Perceptions of authority and leadership: A cross-national, cross-generational investigation. Manag New Workforce Int Perspect Millenn Gener. 2012;281-306.

22. Hinote C, Sundvall TJ. Leading millennials: An approach that works. AIR UNIV MAXWELL AFB AL AIR FORCE RESEARCH INST; 2015.

23. Sharma N, Lau CS, Doherty I, Harbutt D. How we flipped the medical classroom. Med Teach. 2015;37(4):327-330.

24. Hernandez RM. Impact of ICT on Education: Challenges and Perspectives. $J$ Educ Psychol-Propos Represent. 2017;5(1):337-347.

25.Herrington J, Reeves TC, Oliver R. Authentic learning environments. En: Handbook of research on educational communications and technology. Springer; 2014. p. 401-412.

26. Pizzolato JE, Hicklen S. Parent involvement: Investigating the parent-child relationship in millennial college students. J Coll Stud Dev. 2011;52(6):671-686.

27. Dyrbye $\mathbf{L}$, Shanafelt $\mathbf{T}$. A narrative review on burnout experienced by medical students and residents. Med Educ. 2016;50(1):132-149.

28. Boelens R, De Wever B, Voet M. Four key challenges to the design of blended learning: A systematic literature review. Educ Res Rev. 2017;22:1-18.

29. Huang P-H, Haywood M, O'Sullivan A, Shulruf B. A meta-analysis for comparing effective teaching in clinical education. Med Teach. 2019;1-14.

30. Jang HW, Kim K-J. Use of online clinical videos for clinical skills training for medical students: benefits and challenges. BMC Med Educ. 2014;14(1):56.

31. Brandt BF, Kitto S, Cervero RM. Untying the interprofessional Gordian knot: The national collaborative for improving the clinical learning environment. Acad Med. 2018;93(10):1437-1440.

32. Doukas DJ, McCullough LB, Wear S, Lehmann LS, Nixon LL, Carrese JA, et al. The challenge of promoting professionalism through medical ethics and humanities education. Acad Med. 2013;88(11):1624-1629.

33. Kulkarni M. Medical Internship training-challenges and possible solutions. $J$ Educ Technol Health Sci. 2017;4(1):5-6. 